\title{
Resistência insulínica e aumento do risco cardiovascular: uma revisão narrativa
}

\author{
Insulin resistance and increased cardiovascular risk: a narrative review
}

Resistencia a la insulina y aumento del riesgo cardiovascular: una revisión narrativa

Laryssa de Lacerda Rodrigues ${ }^{1 *}$, Isadora Thamires Pacheco Celestino ${ }^{1}$, Alice Ferraz Campos², Anna Carolina Motta Costa ${ }^{3}$, Ariel Andrade Pinheiro ${ }^{4}$, Bárbara Campolina Oliveira ${ }^{4}$, Edmundo Nunes dos Santos Araújo ${ }^{5}$, Lucas Mendes Custódio ${ }^{4}$, Nathália Carvalho França ${ }^{4}$, Vitor Caldeira Matos ${ }^{4}$.

\section{RESUMO}

Objetivo: Realizar uma revisão bibliográfica a fim de analisar o impacto cardiovascular provocado pela resistência à insulina. Revisão Bibliográfica: Foi realizado um levantamento bibliográfico do período de 1979 a 2020 na base de dados Pubmed. Foram selecionados 20 artigos científicos de revisão de literatura nos quais a resistência insulínica $(\mathrm{RI})$ aparece como fator de risco complementar e independente para 0 desenvolvimento de anormalidades cardiovasculares. Foi observado que as principais alterações vasculares decorrem da relação entre a RI e disfunções endoteliais, inflamatórias e hidroeletrolíticas, as quais culminam primordialmente em Hipertensão arterial sistêmica (HAS). Com relação ao efeito cardíaco, observou-se uma relação entre a $\mathrm{RI}$ e alterações da contratilidade e do metabolismo dos cardiomiócitos, resultando principalmente em distúrbios de ejeção e Insuficiência Cardíaca (IC). Considerações finais: A relação entre RI e DCV se mostrou de suma importância, sendo assim, de interesse à prática clínica no desenvolvimento de terapêuticas e propedêuticas que perpassam estes conhecimentos fisiopatológicos.

Palavras-chave: Risco cardiovascular, Resistência insulínica, Insuficiência cardíaca.

\begin{abstract}
Objective: Conduct a literature review in order to analyze the cardiovascular impacto of insulin resistance. Literature Review: A bibliographic survey from 1979 to 2020 was conducted in the Pubmed database. Twenty scientific literature review articles were selected in which IR appears as a complementary and independent risk factor for the development of cardiovascular abnormalities.It was observed that the main vascular alterations result from the relationship between IR and endothelial, inflammatory and hydroelectrolytic dysfunctions, which culminate primarily in systemic arterial hypertension (SAH). Regarding the cardiac effect, a relationship was observed between IR and changes in contractility and cardiomyocyte metabolism, resulting mainly in ejection disorders and Heart Failure (HF). Final considerations: The relationship between IR and CVD proved to be of paramount importance, thus being of interest to clinical practice in the development of therapies and propedeutics that permeate this pathophysiological knowledge.
\end{abstract}

Keywords: Cardiovascular risk, Insulin resistance, Heart failure.

\footnotetext{
1 Pontifícia Universidade Católica de Minas Gerais (PUC-MG), Betim - MG.

*E-mail: laryssadelacerdarod@gmail.com

2 Universidade Federal de Viçosa (UFV), Viçosa - MG.

${ }^{3}$ Universidade Federal de Ouro preto (UFOP), Ouro Preto - MG.

${ }^{4}$ Centro universitário de Belo Horizonte (UNIBH), Belo Horizonte - MG.

${ }^{5}$ Universidade José do Rosário Vellano (UNIFENAS), Belo Horizonte - MG.
} 


\section{RESUMEN}

Objetivo: Realizar una revisión de la literatura con el fin de analizar el impacto cardiovascular de la resistencia a la insulina. Revisión Bibliográfica: Se realizó una encuesta bibliográfica de 1979 a 2020 en la base de datos Pubmed. Se seleccionaron veinte artículos de revisión de la literatura científica en los que el IR aparece como un factor de riesgo complementario e independiente para el desarrollo de anomalías cardiovasculares. Se observó que las principales alteraciones vasculares son el resultado de la relación entre la IR y las disfunciones endoteliales, inflamatorias e hidroelectrolíticas, que culminan principalmente en la hipertensión arterial sistémica (SAH). En cuanto al efecto cardíaco, se observó una relación entre IR y cambios en la contractilidad y el metabolismo de los cardiomiocitos, lo que resulta principalmente en trastornos de eyección e insuficiencia cardíaca (HF). Consideraciones finales: La relación entre IR y ECV resultó ser de suma importancia, siendo así de interés para la práctica clínica en el desarrollo de terapias y propedéuticas que impregnan este conocimiento fisiopatológico.

Palabras clave: Riesgo cardiovascular, Resistencia a la insulina, Insuficiencia cardíaca.

\section{INTRODUÇÃO}

As doenças cardiovasculares (DCV) são um grupo de doenças composto primordialmente por doenças da coronária, processos isquêmicos do coração, acidentes vasculares cerebrais (AVC) e hemorragias cerebrais (HAMAGUCHI E, et al., 2007). Mundialmente, apesar das melhorias na qualidade de vida e do controle da morbimortalidade em geral, observou-se nos últimos anos, um crescimento no número total de casos, que foi motivado, principalmente, pelo envelhecimento e pelo adoecimento da população. Em concordância com esse panorama, segundo a OMS, aproximadamente 23.6 milhões de pessoas morrerão por DCV no ano de 2030, segundo projeções. (BALAKUMAR P, et al., 2016).

Em âmbito nacional essa tendência também prevalece. No Brasil, as doenças cardiovasculares são responsáveis por $27,7 \%$ dos óbitos, atingindo $31,8 \%$ quando são excluídos os óbitos por causa externas, sendo consideradas a principal causa de mortalidade e, por isso, importante fonte de gastos para os sistemas de saúde (MASSA KH, et al., 2019).

Paralelamente, a RI, estado definido pela redução ou ausência da sensibilidade do organismo à ação da insulina, está fortemente relacionada ao aparecimento de doenças cardiovasculares, visto que uma patologia predispõe o aparecimento da outra, sendo o próprio processo patológico vigente no organismo do indivíduo com $\mathrm{RI}$ o responsável pelo desenvolvimento da DCV.

Essa ação ocorre, pelo fato da RI predispor a formação e estabilidade de placas ateromatosas, o aumento do risco de desenvolvimento de IC, a interferência na relação LDL/HDL no organismo e a modificação da oxidação dos ácidos graxos, aumentando o acúmulo destes nos tecidos corporais (SIQUEIRA AFA, 2007; BERTSCH RA e MERCHANT MA, 2015).

Sendo assim, esse é um importante fator que contribui para o aumento da mortalidade por DCV, entrando em concordância com os dados epidemiológicos apresentados nesse artigo, sendo que a perspectiva é de um aumento significativo dos casos de Diabetes Mellitus (DM) nos próximos anos. Estudos apontam que entre 2010 e 2030, concomitantemente ao aumento da obesidade, haverá um aumento nos casos de diabetes entre adultos (NOVO G, et al., 2016).

Dentro do grupo de DCV, se destaca a Insuficiência Cardíaca (IC), doença caracterizada principalmente pela redução da capacidade funcional do coração, com redução de sua fração de ejeção, resultando em sintomas e sinais de baixo débito cardíaco e de congestão pulmonar ou sistêmica. Dessa forma podem surgir sintomas e sinais variáveis de acordo com a gravidade do quadro, como dispnéia, fadiga, edema periférico, ascite, hepatomegalia e taquicardia. (ROHDE LE, et al., 2018). 
É importante ressaltar que a IC é uma das formas de DCV mais comuns, e se tornou a principal causa de mortalidade, de hospitalização e de gastos com cuidados de saúde em indivíduos acima de 65 anos .Possui uma prevalência de $1 \%$ a $2 \%$ da população adulta mundial e é descrita na literatura como uma doença sistêmica multifatorial, em que após uma lesão cardíaca, mecanismos estruturais, neuro-hormonais, celulares e moleculares são ativados de forma compensatória à deficiência deste órgão.

No entanto, mesmo que a finalidade dessas adaptações cardiovasculares sejam a manutenção da função cardíaca, surgem algumas consequências como: sobrecarga de volume, aumento do tônus simpático e alterações da circulação, ocasionando por fim a sintomatologia da IC (FILARDI PP, et al., 2015; TANAI E e FRANTZ S, 2016).

Segundo a atualização de 2019 da Diretriz de Prevenção Cardiovascular da Sociedade Brasileira de Cardiologia, apesar do crescente avanço terapêutico, a IC é considerada uma patologia grave com uma sobrevida de apenas $35 \%$ após 5 anos. Dessa forma é importante conhecer e se possível intervir nos fatores de risco para seu desenvolvimento.

De acordo com a American Heart Association (AHA) e a Sociedade Brasileira de Cardiologia (SBC) são considerados fatores de risco para IC pessoas portadoras de Hipertensão Arterial Sistêmica (HAS), angina, diabetes, alcoolismo, tabagismo, obesidade, sedentarismo, dislipidemia, dieta hiperssódica, Infarto Agudo do Miocárdio (IAM), Hipertrofia Ventricular Esquerda (HVE) e valvulopatia cardíaca (BERTOLIN D, et al., 2019).

Paralelamente, aos casos de RI, a desproporção entre a quantidade de insulina produzida pelo pâncreas e sua atividade no organismo provoca um desequilíbrio homeostático da glicose e de outros metabólitos, o que faz com que a RI seja um dos principais pilares da síndrome metabólica (SM). Esta, por sua vez, é um conjunto de fatores interdependentes observados por aumentarem o risco de pacientes desenvolverem patologias ou eventos cardiovasculares e tromboembólicos.

Entre esses fatores encontram-se, cronicamente, uma pressão arterial sistólica maior ou igual a $130 \mathrm{mmHg}$ ou diastólica maior ou igual a $85 \mathrm{mmHg}$, trigliceridemia maior ou igual a $150 \mathrm{mg} / \mathrm{dL}$, HDL colesterol menor que $40 \mathrm{mg} / \mathrm{dL}$ em homens e $50 \mathrm{mg} / \mathrm{dL}$ em mulheres, circunferências abdominais aumentadas, maior que $102 \mathrm{~cm}$ em homens e $88 \mathrm{~cm}$ em mulheres, além de uma glicemia maior ou igual a $110 \mathrm{mg} / \mathrm{dL}$ caracterizando um perfil de resistência à insulina (BRANDÃO A, et al., 2005).

Em suma, torna-se importante a relação fisiopatológica entre resistência insulínica e doenças cardiovasculares tendo em vista sua coexistência na síndrome metabólica, sua expressividade epidemiológica, os fatores de risco comuns e as possíveis relações de causa e efeito.

O objetivo do presente artigo é apresentar sua interdependência com os seguintes acometimentos: hipertensão arterial sistêmica (HAS) e insuficiência cardíaca (IC). Frente a isso, abrem-se possibilidades ao desenvolvimento de propedêuticas e terapêuticas que aumentem a qualidade de vida dos pacientes e desonerem o sistema de saúde.

\section{REVISÃO BIBLIOGRÁFICA}

A resistência à Insulina (RI) é descrita na literatura como uma condição clínica capaz de comprometer a fisiologia e as funções metabólicas normais de diferentes tecidos e, quando relacionada ao sistema cardiovascular, provoca alterações que são observadas em todos os seus componentes. Uma evidência disso é que pacientes com um alto valor do Homeostasis Model Assessment- Insulin Resistance (HOMA-IR), índice que determina a resistência à insulina e a capacidade funcional das células beta pancreáticas, apresentam o maior risco cardiovascular devido às alterações cardíacas e vasculares também causadas pela resistência à insulina, frequentemente associada à disfunção endotelial e a comorbidades como diabetes mellitus tipo $2 \mathrm{e}$ à obesidade (WESTERGREN HU, et al., 2016) (Quadro 1). 
Quadro 1 - Principais alterações vasculares e cardíacas relacionadas à Resistência à Insulina.

\begin{tabular}{|c|c|}
\hline Autores (ano) & Alterações \\
\hline $\begin{array}{c}\text { Siqueira AFA, et al. (2007); Ormazabal V, et al. } \\
\text { (2018); Madonna R e Caterina R (2012); } \\
\text { Banerjee D, et al. (2014) }\end{array}$ & $\begin{array}{l}\mathrm{Rl}^{* * * * * * *} \text { e níveis de insulina estão associados à } \\
\text { estabilidade de placas ateromatosas e também ao } \\
\text { seu aumento de tamanho, devido à maior deposição } \\
\text { de lipídeos e ao estado pró-inflamatório. }\end{array}$ \\
\hline $\begin{array}{l}\text { Saotome M, et al. (2019); Filardi P, et al. (2015); } \\
\text { Bertsch RA e Merchan MA (2015); Vardeny O, et } \\
\text { al. (2013); Jeppesen J, et al. (2007); Banerjee D, } \\
\text { et al. (2014); Novo G, et al. (2016); Jia G, et al. } \\
\text { (2016); Jia G, et al. (2018); Iglesson E, et al. } \\
\text { (2020) }\end{array}$ & $\begin{array}{l}\mathrm{RI}^{* * * * * *} \text { é fator de risco para } \mathrm{IC}^{*} \text {, presente em mais } \\
\text { de } 60 \% \text { dos portadores, além de predispor a sua } \\
\text { forma congestiva }(\mathrm{ICC})^{\star *} \text {. Baixos níveis de HOMA- } \\
\mathrm{IR}^{* * *} \text { estão associados com menor incidência de IC. }\end{array}$ \\
\hline $\begin{array}{l}\text { Filardi P, et al. (2015); Kozakova M e Palombo C } \\
\text { (2016); Ormazabal V, et al. (2018); Barbalo SM, } \\
\text { et al. (2015); Novo G, et al. (2016); Jia G, et al. } \\
\text { (2016); Jia G, et al. (2018); Patel TP, et al (2016) }\end{array}$ & $\begin{array}{l}\mathrm{RI}^{\star * \star * * * *} \text { como causa de dano celular e disfunção } \\
\text { microvascular associada a rigidez vascular. Isso } \\
\text { ocorre devido a redução dos níveis de óxido nítrico, } \\
\text { provocando vasoconstrição e inflamação. }\end{array}$ \\
\hline $\begin{array}{l}\text { Souza ALL, et al. (2016); Ormazabal V, et al. } \\
\text { (2018); Colaço AL, et al. (2006); Patel TP, et al } \\
\text { (2016) }\end{array}$ & $\begin{array}{l}\text { A RI estimula elevação de angiotensina 2, endotelina } \\
\text { e vasopressina. Portanto, níveis do HOMA-IR }{ }^{\star * *} \\
\text { estão diretamente relacionados com o descontrole da } \\
\text { pressão arterial, devido a vasoconstrição e aumento } \\
\text { da retenção de sódio. }\end{array}$ \\
\hline $\begin{array}{l}\text { Oliveira JS, et al. (2020); Bertsch RA e Merchan } \\
\text { MA (2015); Reaven G, et al. (2012) }\end{array}$ & 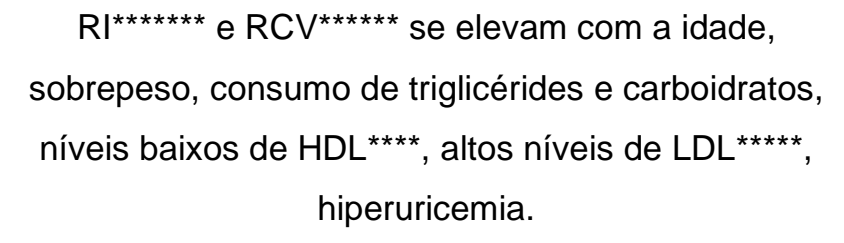 \\
\hline Barbalo SM, et al. (2015) & $\begin{array}{l}\text { Obesidade e síndrome metabólica se associam à } \\
\text { disfunção endotelial e à inflamação, causadas pela } \\
\qquad \mathrm{R}^{\star \star \star \star \star \star \star} \text {. }\end{array}$ \\
\hline
\end{tabular}

Legenda: ${ }^{*} \mathrm{IC}=$ Insuficiência cardíaca; ${ }^{* *} \mathrm{ICC}=$ Insuficiência cardíaca congestiva; ${ }^{* * *} \mathrm{HOMA}-\mathrm{IC}=$ Homeostasis Model Assessment- Insulin Resistance; ${ }^{* * *} \mathrm{HDL}=$ High density lipoprotein; ${ }^{* * * *} \mathrm{LDL}=$ low density lipoprotein; ${ }^{* \star * \star * *} \mathrm{RCV}=$ Risco Cardiovascular; ${ }^{* \star * * * *} \mathrm{Rl}=$ Resistência insulínica.

Fonte: Rodrigues LL, et al., 2020.

Em vista disso, com base na fisiopatologia desta condição clínica é relevante discutir as alterações vasculares e cardíacas que de maneira distinta, atuam juntas na disfunção metabólica e fisiológica do sistema cardiovascular (Figura 1). 
Figura 1 - Fluxograma dos efeitos cardiovasculares da resistência à insulina.

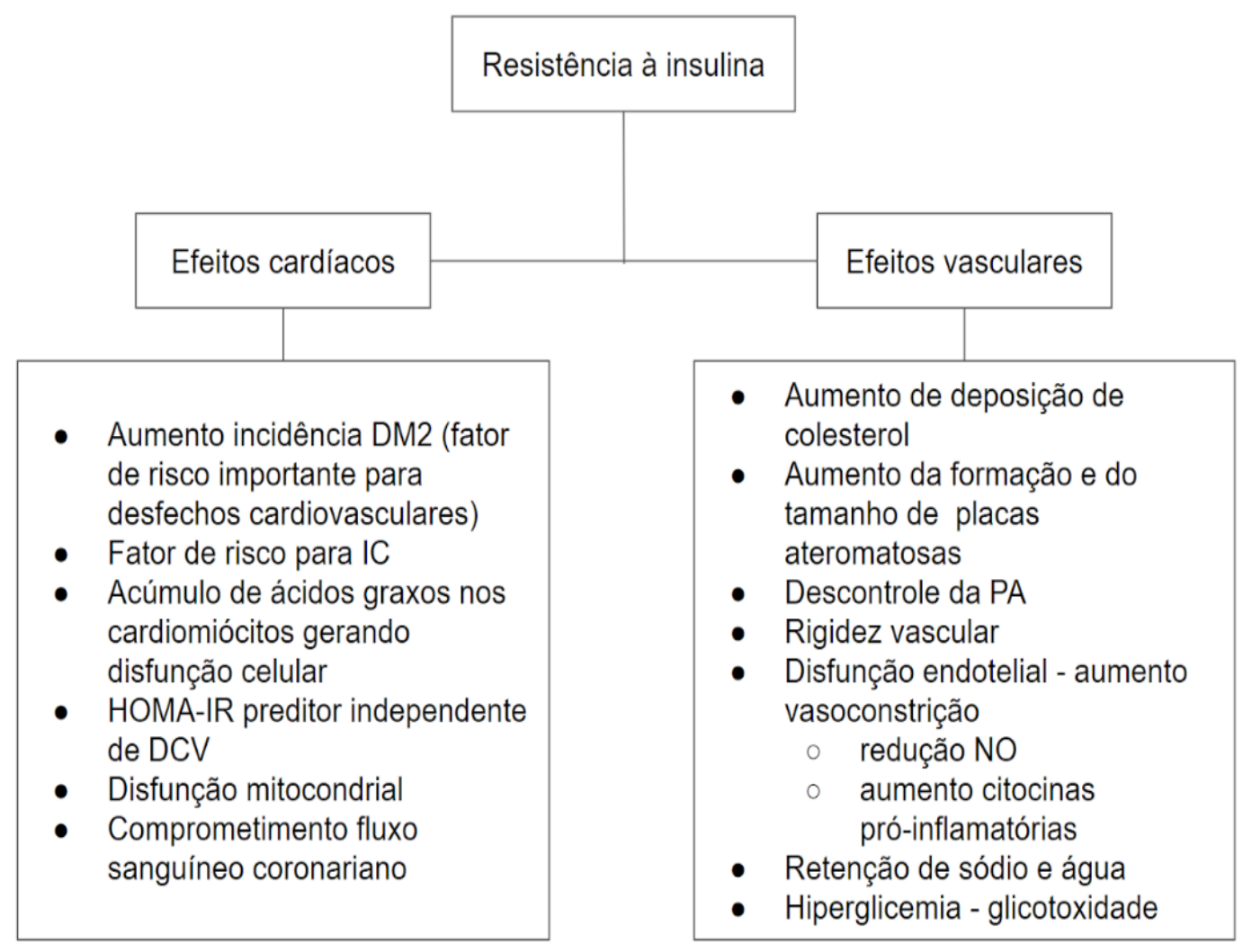

Fonte: Rodrigues LL, et al., 2020.

Em âmbito vascular, a RI é uma das causas primárias de disfunção endotelial, cujos principais componentes são: desequilíbrio de mediadores do tônus vascular, alteração da agregação plaquetária, distúrbios de coagulação e fibrinólise. Todos estes efeitos estão associados a diferentes processos patológicos que acometem os vasos sanguíneos como aterosclerose, hipertensão arterial, dislipidemia e Diabetes Mellitus (COLAÇO AL, et al., 2006).

Paralelamente nos quadros de resistência ocorre uma diminuição da biodisponibilidade de óxido nítrico (NO), acúmulo de espécies reativas de oxigênio (EROs) e aumento da expressão de fatores pró-inflamatórios e pró-coagulantes que promovem a adesão de leucócitos às células endoteliais. Essas alterações são marcadoras de disfunção endotelial, o que contribui também para o aumento do risco cardiovascular (RCV), já que este é mais elevado em quadros de aterosclerose, instabilidade das placas aterosclerótica, acumulação de macrófagos e hipertensão (ORMAZABAL V, et al., 2018).

Com relação ao nível celular a insulina atua de maneira a criar um sistema compensatório de vasoconstrição e vasodilatação que age sobre o endotélio estimulando a produção de óxido nítrico, agente vasodilatador, atenuador de resposta inflamatória e inibidor da proliferação de células musculares lisas. Esse processo ocorre via fosfatidilinositol 3-cinase (PI3K), dependente da sinalização de insulina e contrabalanceia a secreção e ação de endotelina-1, um vasoconstritor, pró-inflamatório que produz efeito mitótico em células musculares lisas, via Mitogen Activated Protein Kinases (MAPK). No entanto, em um estado patológico de RI e de hiperinsulinemia, há um prejuízo na via de produção de NO, possivelmente devido a uma resposta alterada e ineficaz no músculo liso, ao passo que a via da MAPK, uma via de ativação compensatória à diminuição do efeito de óxido nítrico, continua com sua atividade inalterada ou até mesmo aumentada. Consequentemente a via da MAPK libera citocinas vasoconstritoras e inflamatórias, que aumentam o tônus vascular e a retenção de sódio e água, aumentando por fim a pressão arterial do indivíduo (MUNIYAPPA R e SOWERS JR, 2014). 
As seguintes disfunções endoteliais causadas pelo desequilíbrio entre a produção de NO e moléculas vasoconstritoras (Angiotensina II, Endotelina-1, prostanóides): vasorreatividade anormal, desequilíbrio da divisão de células musculares lisas e aumento de moléculas de adesão endotelial, permitem caracterizar a via da MAPK como um possível fator ateratogênico, uma vez que a endotelina-1 é uma das principais substâncias mitogênicas e vasoconstritoras produzidas pelo endotélio, além de que juntamente à este desequilíbrio ocorre aumento do estresse oxidativo, maior migração celular e liberação de fatores próinflamatórios e pró-trombóticos. Sendo assim, é uma questão importante saber se a via está atenuada em estados de resistência à insulina através de biomarcadores de inflamação e de estresse oxidativo (CARVALHO MH, et al., 2006; MUNIYAPPA R e SOWERS JR, 2014).

A RI além de estar relacionada à redução da produção de NO, gera retenção de sódio, o que propicia o aumento da pressão arterial pela consequente retenção de água e aumento do volume de sangue circulante. Além do aumento a pressão arterial, a RI está relacionada ao aumento da expressão e da concentração plasmática de citocinas pró-inflamatórias, levando o organismo a um estado de inflamação crônica. A condição pró-inflamatória sugere ligação entre resistência à insulina e disfunção endotelial no estágio inicial do processo de aterosclerose, em indivíduos obesos e em pacientes com Diabetes Mellitus 2 (DM2) (COLAÇO AL, et al., 2006).

Paralelamente, a insulina também exerce efeito sobre o colesterol. Fomenta o mecanismo produtor de colesterol, através do aumento da atividade da enzima que controla esse mecanismo, a 3-hidroxi-3-methylglutaril-CoA redutase (HMG-CoA). Os mecanismos fisiopatológicos pelos quais a hiperinsulinemia resulta numa aceleração da aterotrombogênese são: a oxidação do LDL, que adere a parede arterial, favorecendo a agregação plaquetária, as instabilidades das placas no paciente dislipidêmico e a consequente inflamação decorrente desse processo. Juntamente a isto, observa-se uma redução da atividade do HDL, culminando assim com o aumento de gordura na placa ateromatosa (COLAÇO AL, et al., 2006).

Além disso, sabe-se que a RI estava associada a um aumento da pró-insulina circulante, que por sua vez atua aumentando a síntese do inibidor da ativação do plasminogênio (PAI-1). Diante dessa maior inibição sobre a ação do plasminogênio, tem-se um prejuízo na fibrinólise, resultando em placas ateromatosas mais estáveis. Ademais, a hiperinsulinemia resulta em um aumento da migração e da atividade mitogênica de células musculares lisas nas artérias, culminando com um crescimento do número dessas células em placas ateromatosas e uma consequente progressão do tamanho da placa. Sendo assim, a maior formação de placas, associada à formação de placas de tamanhos maiores possui forte relação com o aumento da pressão arterial e do esforço realizado pelo miocárdio para propelir o sangue (SIQUEIRA AF, et al., 2006).

O acúmulo de tecido adiposo visceral (IVA) está ligado a uma maior incidência de resistência à insulina, DM2 e maior risco de doença cardiovascular. Dessa forma, pessoas obesas com índice de massa corporal (IMC) igual ou maior que $30 \mathrm{~kg} / \mathrm{m}^{2}$ tem risco aumentado para DCV e RI quando comparada com pessoas de IMC normal. Ademais, fatores como inflamação decorrente do acúmulo de lipídios, estresse oxidativo, efeito inibitório da oxidação de ácidos graxos na oxidação da glicose e secreção de adipocitoquinas têm sido associados ao desenvolvimento de RI local e sistêmica (ORMAZABAL V, et al., 2018).

Quando há excesso de calorias de forma crônica, os adipócitos sofrem hipertrofia e hiperplasia ocasionando o aumento da massa de gordura visceral. Ocorre uma resposta inflamatória no tecido adiposo devido a liberação fatores quimiotáticos como o Fator de Necrose Tumoral alfa (TNF- $\alpha$ ) e o Monocyte chemoattractant protein- 1 (MCP-1). O MCP-1 é responsável pela entrada dos monócitos no tecido adiposo visceral e diferenciação dos monócitos em macrófagos - que aumentam a lipólise através da secreção aumentada de TNF- $\alpha$ e consequente diminuição do transportador de glicose (GLUT4), além da biossíntese de triglicérides fazendo com que o IVA armazene adipócitos, acarretando um aumento dos triglicérides.

Os fatores supracitados podem acarretar uma deposição lipídica ectópica de ácidos graxos no pâncreas, vasos sanguíneos renais, fígado, músculo esquelético e coração - que leva a um aumento do tecido adiposo epicárdico (TEA). Em meio a isso, o aumento do TAE pode provocar a hipertrofia de ventrículos, disfunções contráteis, fibroses e função do ventrículo esquerdo (VE) prejudicada (SOUMYALEKSHMI N, et al., 2017). 
Também é importante destacar que a deposição lipídica hepática e miocárdica está correlacionada à resistência à insulina (RI), ao risco cardiovascular e ao aumento da capacidade oxidativa hepática com estresse oxidativo e uma deficiência mitocondrial. Esta disponibilidade lipídica promove disfunção cardíaca induzida por isquemia e diminui a eficiência mitocondrial do miocárdio. Com o aumento da disponibilidade lipídica e a RI, o organismo fica mais suscetível à disfunção cardíaca induzida pela isquemia (JELENIK T, et al., 2018).

Com o estudo de Framingham, foi demonstrado que a resistência insulínica é um fator de risco direto para insuficiência cardíaca. Isso porque independente da coexistência de outros fatores de risco cardiovasculares, foi observado que a presença exclusiva do Diabetes Mellitus tipo 2 (DM2) tem impacto no desenvolvimento da doença. Observou-se que o aumento de $1 \%$ na $\mathrm{HbA} 1 \mathrm{c}$ aumenta em $8 \%$ o risco de IC, independente de outros fatores como pressão arterial, idade, IMC ou doença coronariana prévia. Já a diminuição de 1\% na $\mathrm{HbA} 1 \mathrm{c}$ diminuiu em $16 \%$ o risco de IC, demonstrando assim o fator bidirecional de sua influência (JIA G, et al., 2015).

O miocárdio utiliza glicose e ácidos graxos (AG) como substrato energético para suas fibras. Os ácidos graxos são internalizados através das enzimas CD36 e FAT e a glicose através da GLUT4, estimulada pela insulina, que se direcionam para o sarcolema do miócito quando o plasma apresenta riqueza desses substratos. Este mecanismo de produção energética cardíaca em condições normais apresenta uma flexibilidade metabólica, dando preferência a glicose ou ácidos graxos como substrato de acordo com as necessidades que o corpo apresenta. (JIA G, et al., 2015; JIA G, et al. 2018).

Em contrapartida, em um estado de resistência insulínica a flexibilidade metabólica é prejudicada, já que o GLUT4 não é externalizado e apenas CD36 e FAT são direcionadas para o sarcolema, internalizando apenas ácidos graxos para as células miocárdicas. $O$ excesso de ácidos graxos promove alterações que custam ao miocárdio maior consumo de oxigênio e consequente perda na resposta das fibras aos estímulos elétricos. Com a evolução da patologia, o estresse metabólico-oxidativo aumenta no músculo cardíaco, diminuindo sua contratilidade e gerando apoptose de miócitos. (JIA G, et al., 2015; JIA G, et al. 2018).

Um fator que se mostrou determinante na resistência cardíaca à insulina e suas demais consequências foi a disfunção mitocondrial dos cardiomiócitos. A disfunção mitocondrial induz um aumento na produção de radicais livres reduzindo com isso o potencial de membrana mitocondrial, diminuindo a atividade do complexo da cadeia transportadora de elétrons e prejudicando a biogênese mitocondrial.

Diante dessa alteração na função das mitocôndrias foi possível perceber um aumento do acúmulo de lipídeos intracelulares, os quais por sua vez produzem resíduos de serina fosforilada no IRS-1 (Receptor de insulina), que neste estado se torna inativo, além de uma supressão da translocação do GLUT-4. Sendo assim, os cardiomiócitos passam a produzir menos ATP e apresentam uma capacidade de remodelamento cardíaco reduzida, podendo culminar com um defeito de ejeção ou mesmo uma IC (SÃO TOME M, et al., 2019).

Em um estudo sobre a Síndrome Metabólica pacientes com RI e IC associados apresentavam formas mais agressivas de disfunção ventricular esquerda e maior índice de mortalidade do que quando comparados com pacientes com IC e sem RI. Além disso, a resistência à insulina se mostrou presente em mais de $60 \%$ dos pacientes com IC. Essas correlações podem ser justificadas por um aumento da ativação do sistema neuro hormonal e do sistema simpático, resultando em um relaxamento ineficiente do coração, além de provocar alterações nos mecanismos de funcionamento das proteínas de contratilidade (FILARDI P, et al., 2015).

Pacientes com insuficiência cardíaca têm maiores níveis de insulina em jejum e maior prevalência de resistência à insulina. A resistência à insulina leva a anormalidades funcionais e estruturais no coração, como o aumento do volume do átrio, ligado à disfunção diastólica, e também, um aumento do Ventrículo Esquerdo, considerado marcador de eventos cardiovasculares como a fibrilação atrial, acidente vascular cerebral, insuficiência cardíaca e óbito (BANERJEE D, et al., 2015). 
Por si só, a hiperglicemia constante gera aumento das espécies reativas de oxigênio circulantes no sangue, as quais são responsáveis pela degradação de moléculas de DNA e inibição da enzima G3PDH, culminando com o excesso de produtos finais da glicação avançada. Esses produtos, por fim, estimulam o acúmulo de colágeno no miocárdio gerando fibrose tecidual e diminuição da sua complacência (JIA G, et. al, 2015; ORMAZABAL V, et al., 2018).

A RI, assim como a hiperinsulinemia e a hiperglicemia são fatores de risco independentes para o desenvolvimento da cardiomiopatia diabética, caracterizada por anormalidades do relaxamento miocárdico e por IC. Parte da fisiopatologia se deve por uma falha na sinalização da insulina nos cardiomiócitos que culmina com o aumento da concentração intracelular de cálcio, além de uma redução de produção de NO que eleva a razão N2B/N2BA das isomorfas da tinina o que torna o sarcômero mais rígido. (JIA G, et. al., 2018).

Outro fator importante no desenvolvimento dessa IC é o déficit na circulação coronariana devido aos baixos níveis de óxido nítrico circulantes. Este fator age reduzindo a ativação da MLCP (myosin light chain phosphatase) e da MLCK (myosin light chain kinase). Sem a atuação do NO, a ativação dessas enzimas leva ao aumento dos níveis de Ca2+ intracelular nas células musculares lisas das coronárias e as induzem a um estado de constrição, diminuindo a eficiência da irrigação do tecido miocárdico (VARDENY O, et al., 2013; JIA G, et al., 2018).

Com relação à prática clínica ainda não existem critérios diagnósticos a respeito da resistência cardíaca à insulina, no entanto, estudos mostraram que tratamentos com antidiabéticos continham efeitos diferentes quanto à eficácia para redução da glicemia e melhora da IC, sendo que alguns antidiabéticos até mesmo levaram a uma piora do quadro de IC. A Metformina que é o atual tratamento de primeira linha para pacientes com DM2 e DCV, tem seu uso respaldado por ser um fármaco que apresentou menor risco de hospitalização. A exemplo dessa diferença de efeitos entre fármacos antidiabéticos se tem os fármacos da família agonista de PPAR- $\chi$, que por aumentarem a retenção de sódio e água, melhoram a glicemia, mas podem promover uma piora do quadro cardíaco. Em contrapartida tem-se os fármacos da família agonista do receptor GLP-1, muito presente no miocárdio, que além de promover uma melhora na glicemia, melhora a contratilidade cardíaca e com isso reduz a mortalidade e as hospitalizações por conta da DCV. Diante disso, torna-se relevante mais estudos a respeito de possíveis marcadores de resistência cardíaca à insulina para aumentar a eficácia das práticas propedêuticas e terapêuticas (SAOTOME M, et al., 2019).

\section{CONSIDERAÇÕES FINAIS}

A insulina é um hormônio essencial para a homeostase do corpo humano. O desequilíbrio de seus níveis pode levar ao estado de RI. A hiperglicemia, dislipidemia e o estresse oxidativo são as principais causas da RI. Estudos recentes relacionam este estado metabólico com mecanismos fisiopatológicos de dano celular e vascular, causando desfechos cardiovasculares a longo prazo. $O$ dano nas funções endoteliais, o aumento da pressão arterial e o comprometimento do metabolismo dos cardiomiócitos intensifica e corrobora com a fisiopatologia das DCV, demonstrando sua direta relação com a RI. Por conseguinte, são necessários mais estudos para solidificar essa relação, devido a alta epidemiologia das DCV, objetivando oferecer aos pacientes terapêuticas e propedêuticas aprimoradas e mais eficazes.

\section{REFERÊNCIAS}

1. BALAKUMAR P, et al. Prevalence and prevention of cardiovascular disease and diabetes mellitus. Elsevier, 2016; 113:600-609.

2. BANERJEE D, et al. Insulin resistance and risk of incident heart failure cardiovascular health study. Circulation: Heart Failure, 2013; 6(3):364-70.

3. BARBALHO SM, et al. Metabolic syndrome, atherosclerosis and inflammation: An inseparable triad? Jornal Vascular Brasileiro, 2015; 14(4):319-27.

4. BERTOLIN D, et al. Atualização da Diretriz de Prevenção Cardiovascular da Sociedade Brasileira de Cardiologia. Arq Bras Cardiol, 2019;113(4):787-891.

5. BERTSCH RA, MERCHANT MA. Study of the Use of Lipid Panels as a Marker of Insulin Resistance to Determine Cardiovascular Risk. The Permanente Journal, 2015; 19(4):4-10 
6. BRANDÃO A, et al. I Diretriz Brasileira de Diagnóstico e Tratamento da Síndrome Metabólica. Arq. Bras. Cardiol, 2005; 84:1678-4170.

7. CARMO MM, et al. Será a síndrome metabólica um marcador de prognóstico em doentes com elevado risco cardiovascular? Um estudo de coorte a longo-prazo. Revista Portuguesa de Cardiologia, 2019; 38(5):325-332.

8. DE CARVALHO MH, et al. Citocinas, disfunção endotelial e resistência à insulina. Arquivos Brasileiros de Endocrinologia, 2006; 50(2):304-12.

9. DE ANDRADE MIS, et al. Prevalence of insulin resistance and association with metabolic risk factors and food consumption in adolescents. Revista Paulista de Pediatria, 2020; 38:e2019016.

10. DE MORAIS PRS, et al. Correlation of insulin resistance with anthropometric measures and blood pressure in adolescents. Arquivos Brasileiros de Cardiologia, 2016; 106(4):319-26.

11. FILARDI $P$, et al. The role of metabolic syndrome in heart failure. European Heart Journal, 2015; 36(39):2630-4

12. GINSBERG HN, et al. Insulin resistance and cardiovascular disease. Journal of Clinical Investigation, 2000; 106(4):453-8.

13. HAMAGUCHI E, et al.Nonalcoholic fatty liver disease is a novel predictor of cardiovascular disease. World $J$ Gastroenterol, $2007 ; 13(10): 1579-1584$.

14. INGELSSON E, et al. Insulin resistance and risk of congestive heart failure. Journal of the American Medical Association, 2005; 294(3):334-41.

15. JELENIK T, et al. Insulin resistance and vulnerability to cardiac ischemia. Diabetes, 2018; 67(12):2695-702.

16. JEPPESEN J, et al. Insulin Resistance, the Metabolic Syndrome, and Risk of Incident Cardiovascular Disease.. Journal of the American College of Cardiology, 2007; 49(21):2112-9.

17. JIA G, et al. Insulin resistance and hyperinsulinaemia in diabetic cardiomyopathy. Nature Reviews Endocrinology, 2016; 12(3):144-53.

18. JIA G, et al. Diabetic cardiomyopathy: a hyperglycaemia- and insulin-resistance-induced heart disease. Cardiovascular Diabetology, 2018; 61(1):21-8.

19. KANNEL WB, MCGEE DL. Diabetes and cardiovascular risk factors: The Framingham study. Circulation, 1979; 59(1):8-13.

20. KOZAKOVA M, PALOMBO C. Diabetes mellitus, Arterialwall, and cardiovascular risk assessment. International Journal of Environmental Research and Public Health, 2016; 13(2).

21. KUWABARA M, et al. The Relationship between Fasting Blood Glucose and Hypertension. American Journal of Hypertension, 2019; 32(12):1143-5.

22. MADONNA R, DE CATERINA R. Atherogenesis and Diabetes: Focus on Insulin Resistance and Hyperinsulinemia. Revista Española de Cardiología, 2012; 65(4):309-13.

23. MASSA KH, et al. Analysis of the prevalence of cardiovascular diseases and associated factors among the elderly, 2000-2010. Ciênc. saúde coletiva, 2019; 24:1678-4561

24. MUNIYAPPA R, SOWERS JR. Role of insulin resistance in endothelial dysfunction. Reviews in Endocrine and Metabolic Disorders, 2013; 14(1):5-12.

25. NOVO G, et al. Impact of insulin resistance on cardiac and vascular function. International Journal of Cardiology, 2016; 221:1095-9.

26. ORMAZABAL V, et al. Association between insulin resistance and the development of cardiovascular disease. Cardiovascular Diabetology, 2018; 17(1):1-14.

27. SALVETTI A, et al. The Inter-Relationship between Insulin Resistance and Hypertension. Drugs, 1993; 46(2):149-59.

28. SAOTOME M, et al. Cardiac insulin resistance in heart failure: The role of mitochondrial dynamics. International Journal of Molecular Sciences, 2019; 20(14).

29. PATEL TP, et al. Insulin resistance: an additional risk factor in the pathogenesis of cardiovascular disease in type 2 diabetes. Heart Failure Reviews, 2016; 21(1):11-23.

30. REAVEN G. Insulin resistance and coronary heart disease in nondiabetic individuals. Arteriosclerosis, Thrombosis, and Vascular Biology, 2012; 32(8):1754-9.

31. ROHDE LE, etal. Diretriz Brasileira De Insuficiência Cardíaca Crônica e Aguda.Arq BrasCardiol,2018;111(3):436-539.

32. SIQUEIRA AFA, et al. Doença cardiovascular no diabetes mellitus: Análise dos fatores de risco clássicos e nãoclássicos. Arquivos Brasileiros de Endocrinologia \& Metabologia, 2007; 51(2):257-67.3456.

33. VARDENY O, et al. Insulin Resistance and Incident Heart Failure. The ARIC Study (Atherosclerosis Risk in Communities). JACC: Heart Failure, 2013; 1(6):531-6.

34. WESTERGREN HU, et al. Insulin resistance, endothelial function, angiogenic factors and clinical outcome in nondiabetic patients with chest pain without myocardial perfusion defects. Cardiovascular Diabetology, 2016; 15(1):1-12. 